\title{
A Warehouse Imperfect Fuzzified Production Model with Shortages under Inflationary Conditions
}

\author{
S. R. Singh, ${ }^{1}$ Shalini Jain, ${ }^{2}$ and Sarla Pareek ${ }^{2}$ \\ ${ }^{1}$ Department of Mathematics, D.N. College, Meerut 250001, India \\ ${ }^{2}$ Centre for Mathematical Sciences, Banasthali University, Banasthali, Rajasthan 304022, India \\ Correspondence should be addressed to S. R. Singh, shivrajpundir@gmail.com
}

Received 30 April 2012; Revised 17 October 2012; Accepted 9 November 2012

Academic Editor: S. Dempe

Copyright (C) 2012 S. R. Singh et al. This is an open access article distributed under the Creative Commons Attribution License, which permits unrestricted use, distribution, and reproduction in any medium, provided the original work is properly cited.

\begin{abstract}
We develop a two-warehouse production model with imperfect items. Production rate is taken as the linear combination of on-hand inventory and demand, while demand rate is taken as function of time. Most of the researchers consider that the production rate is independent from the demand rate. In this paper we assume production rate as being dependent on the demand rate, and this assumption is more realistic. Shortages are allowed and partially backlogged with time-dependent backlogging rate. Due to different preservation facilities we consider that the deterioration rate is time dependent in own warehouse $(\mathrm{OW})$ and Weibull distribution deterioration in rented warehouse (RW). Holding cost in RW is greater than in OW. We developed a fuzzy model with fuzzifying all the costs of the model as triangular fuzzy numbers. The present model is developed in both crisp and fuzzy senses. Finally, numerical example is shown, and sensitivity is also illustrated.
\end{abstract}

\section{Introduction}

One of the weaknesses of some production-inventory models is the unrealistic assumption that all items produced are of good quality. But production of defective units is a natural phenomenon in a production process. Defective items should be treated as a result of imperfect quality production. The effect of an imperfect process on production run time and EPQ was initially studied by Rosenblatt and Lee [1]. In their study, the elapsed time until the process shift was assumed to be exponentially distributed. The optimal production run was found to be shorter than that of classical EPQ model. In recent years, numerous research efforts have been undertaken to extend the work of Rosenblatt and Lee [1]. Kim and Hong [2] extended the work of Rosenblatt and Lee [1] by assuming that elapsed time 
until the process shift was arbitrarily distributed. However, neither of their models took into consideration of any allowable shortages. Chung and Hou [3], however, have generalized the work of Kim and Hong [2] by assuming that shortages were allowed. Nevertheless, the common assumption of all the above-mentioned models was that there were a fixed percentage of defective items produced during the out-of-control period. C. Singh and S. R. Singh [4] developed an imperfect production process with exponential demand rate, Weibull deterioration under inflation.

In some cases, uncertainties can be defined as fuzziness or vagueness, which are characterized by fuzzy numbers of the fuzzy set theory. Zadeh [5] introduced fuzzy set theory to deal with quality-related problems with imprecise demand. Bellman and Zadeh [6] distinguished the difference between randomness and fuzziness by showing that the former deals with uncertainty regarding membership or nonmembership of an element in a set, while the later is concerned with the degree of uncertainty by which an element belongs to a set. Gen et al. [7] expressed the input data by fuzzy numbers, where they used interval mean value concept to solve an inventory problem. Yao and Chiang [8] considered an inventory model with total demand and storing cost as triangular fuzzy numbers. They performed the defuzzification by centroid and signed distance methods. Mondal and Maiti [9] applied genetic algorithms (GAs) to solve a multi-item fuzzy EOQ model. M. K. Maiti and M. Maiti [10] dealt with a fuzzy inventory model with two warehouses under possibility constraints. Mahapatra and Maiti [11] formulated a multi-item, multiobjective inventory model for deteriorating items with stock- and time-dependent demand rate over a finite time horizon in fuzzy stochastic environment. Halim et al. [12] developed a fuzzy inventory model for perishable items with stochastic demand, partial backlogging, and fuzzy deterioration rate. The model is further extended to consider fuzzy partial backlogging factor. Gani and Maheswari [13] discussed the retailer's ordering policy under two levels of delay payments considering the demand and the selling price as triangular fuzzy numbers. They used graded mean integration representation method for defuzzification. Lee and Yao [14] developed an economic production quantity (EPQ) model in which the demand and the production quantity are assumed to be fuzzy. Halim et al. [15] addressed the lot sizing problem in an unreliable production system with stochastic machine breakdown and fuzzy repair time. They defuzzified the cost-per-unit time using the signed distance method. Production of defective items in any manufacturing industry is a natural phenomenon. The number of defectives may have a change from one lot to another that cannot be assessed by a crisp value. Chen and Chang [16] developed a fuzzy economic production quantity (EPQ) model with defective productions that cannot be repaired. In this model, they considered a fuzzy opportunity cost and trapezoidal fuzzy costs under crisp production quantity or fuzzy production.

Since most decision makers think that the inflation does not have significant influence on the inventory policies, the effects of inflation are notconsidered in some inventory models. However, from a financial point of view, an inventory represents a capital investment and must compete with other assets for a firm's limited capital funds. Thus, it is necessary to consider the effects of inflation on the inventory system. Buzacott [17], Bierman and Thomas [18], and Misra [19] investigated the inventory decisions under an inflationary condition for the EOQ model. Chandra and Bahner [20], Moon and Lee [21], Lo et al. [22], and Singh et al. $[23,24]$ are amongst the few who studied the concept of inflation with regard to inventory. Singh and Jain [25] explored a deterministic inventory model for a deteriorating item in an inflation-induced environment. 
In this paper, we assumed the following assumptions.

(i) Production rate is greater than the demand rate.

(ii) Production rate is the linear combination of on-hand inventory and demand rate.

(iii) Demand rate is exponentially an increasing function of time.

(iv) Model is considered for imperfect items.

(v) Deterioration is taken as time dependent for OW, while, Weibull distribution for RW.

(vi) Inflation is also taken in this model.

(vii) Costs are considered as a triangular fuzzy numbers.

(viii) Model is presented in both fuzzy and crisp senses.

\section{Assumptions and Notations}

\subsection{Assumptions}

The following assumptions have been used throughout the study.

(i) The demand rate is exponentially increasing and is represented by $\lambda(t)=\lambda_{0} e^{\delta t}$, where $0 \leq \delta \leq 1$ is a constant inflation rate, and $\lambda_{0}$ is the initial demand rate.

(ii) Lead time is zero, and no replenishment or repair of deteriorated items is made during a given cycle.

(iii) Production rate is the linear combination of on-hand inventory and demand rate at any time subject to

$$
P(t)=[I(t)+\eta \lambda(t)]\left(1-e^{-\Delta t}\right), \quad 0 \leq \Delta \leq 1, \eta>1, P(t)>\lambda(t) .
$$

(iv) Shortages are allowed and the backlogging rate is exponentially, a decreasing function of time when inventory is in shortages, the backlogging parameter $s$ is a positive constant s.t. $0<s<1$.

(v) A single item is considered over the prescribed period $T$ units of time, which is subject to variable deterioration rate.

(vi) The owned warehouse (OW) has a fixed capacity of $W$ units, and the rented warehouse (RW) has unlimited capacity.

(vii) The goods of OW are consumed only after consuming the goods kept in RW.

(viii) The unit inventory costs (including holding cost) per unit time in RW are higher than those in OW.

(ix) Deterioration-rate of the items is considered to be different in different warehouses. In OW time-dependent deterioration rate is $\theta(t)=\theta t$, while in RW Weibull distribution deterioration rate is $\alpha \beta t^{\beta-1}$, where $\alpha, \beta>0, t>0$.

(x) The model is developed in both fuzzy and crisp environment.

(xi) Finite planning horizon is considered. 


\subsection{Notations}

The following notations have been used throughout the study.

$\lambda$ : Demand rate, $\lambda(t)=\lambda_{0} e^{\delta t}$, increases with time, where $\lambda_{0}$ is the initial demand rate and $0 \leq \delta \leq 1$.

$P(t)$ : Production rate $P(t)=[I(t)+\eta \lambda(t)]\left(1-e^{-\Delta t}\right)$, which is the linear combination of on-hand inventory and demand rate at any time and $P(t)>\lambda(t)$, where $0 \leq \Delta \leq 1$, $\eta>1$.

$B$ : Backlogging rate, $B=e^{-s t}$, where the backlogging parameter $s$ is a positive constant s.t. $0<s<1$.

$\theta$ : Deterioration rate, where $\theta=\theta t$ for OW and $\theta=\alpha \beta t^{\beta-1}$ for RW, where $\alpha, \beta>0$, $t>0$.

$\Delta$ : Rate of imperfect production.

$r$ : Inflation rate.

$W$ : Fixed capacity level of OW.

$C_{1}$ : Set up cost per production run.

$C_{\mathrm{RW}}$ : Carrying cost per inventory unit held in RW per unit time.

$C_{\text {OW }}$ : Carrying cost per inventory unit held in OW per unit time.

$\mathrm{C}_{2}$ : Deterioration cost per unit time.

$C_{3}$ : Shortage cost for backlogged items.

$C_{4}$ : Lost sale cost per unit time.

$C_{5}$ : Rework cost per unit time.

$I_{i 1}$ : Inventory level in OW at time $t$ with $t \in\left[0, t_{1}\right]$.

$I_{i 2}$ : Inventory level in RW at time $t$ with $t \in\left[t_{1}, t_{2}\right]$.

$I_{i 3}$ : Inventory level in RW at time $t$ with $t \in\left[0, t_{3}\right]$.

$I_{i 4}$ : Inventory level in OW at time $t$ with $t \in\left[0, t_{4}\right]$.

$I_{i 5}$ : Inventory level in OW at time $t$ with $t \in\left[0, t_{2}+t_{3}\right]$.

$I_{i 6}$ : Inventory level in OW at time $t$ with $t \in\left[0, t_{5}\right]$.

$I_{i 7}$ : Inventory level in OW at time $t$ with $t \in[0, T]$.

$\widetilde{C}_{1}$ : Fuzzy set up cost per unit time.

$\tilde{C}_{2}$ : Fuzzy deterioration cost per unit time.

$\widetilde{C}_{3}:$ Fuzzy shortage cost per unit time.

$\widetilde{C}_{4}$ : Fuzzy lost sale cost per unit time.

$\tilde{C}_{5}$ : Fuzzy rework cost per unit time.

$\widetilde{C}_{\text {Ow: }}$ Fuzzy carrying cost per unit time FOR OW.

$\tilde{C}_{\mathrm{RW}}$ : Fuzzy carrying cost per unit time for RW.

$t_{1}, t_{2}$ : The production periods for OW and RW.

$t_{3}, t_{4}$ : The non production periods.

$t_{5}$ : The shortage period.

$T$ : Total cycle time. 


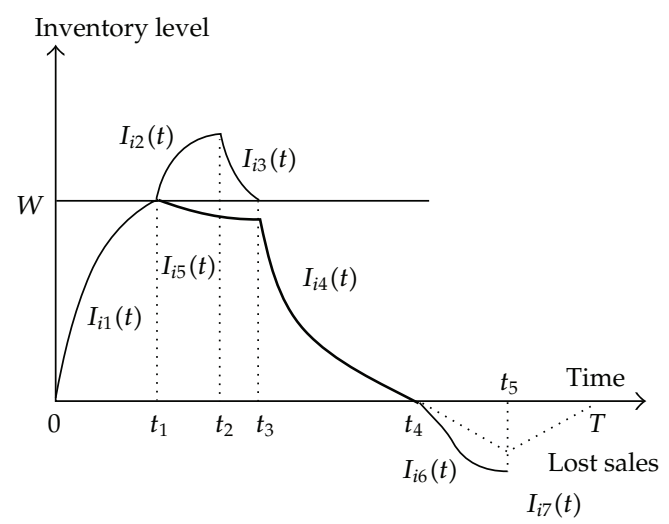

Figure 1: Inventory level in production system for deteriorating items with inflation-induced demand.

\section{Formulation and Solution of the Model}

Figure 1 shows the inventory level during a production cycle in which both OW and RW are used. Initially, the inventory level is zero. The production starts at time $t=0$, and items accumulate from 0 up to $W$ units in $\mathrm{OW}$ and in $t_{1}$ units of time. After time $t_{1}$ any production quantity exceeding $W$ will be stored in RW. After this production stopped and the inventory level in RW begins to decrease at $t_{2}$ and will reach 0 units at $t_{3}$ because of demand and deterioration. The inventory level in OW comes to decrease at $t_{1}$ and then falls below $W$ at $t_{2}+t_{3}$ due to deterioration. The remaining stocks in OW will be fully exhausted at $t_{4}$ owing to demand and deterioration: the inventory becomes zero. At this time shortage starts developing, and at time $t_{5}$ it reaches to maximum shortage level : at this time fresh production starts to clear the backlog by the time $T$. follows:

The differential equations stating the inventory levels within the cycle are given as

$$
\begin{gathered}
\frac{d I_{i 1}(t)}{d t}+\theta I_{i 1}(t)=P(t)-\lambda(t), \quad 0 \leq t \leq t_{1} . \\
\frac{d I_{i 2}(t)}{d t}+\theta I_{i 2}(t)=P(t)-\lambda(t), \quad t_{1} \leq t \leq t_{2} . \\
\frac{d I_{i 3}(t)}{d t}+\theta I_{i 3}(t)=-\lambda(t), \quad t_{2} \leq t \leq t_{3} . \\
\frac{d I_{i 4}(t)}{d t}+\theta I_{i 4}(t)=-\lambda(t), \quad t_{3} \leq t \leq t_{4} . \\
\frac{d I_{i 5}(t)}{d t}+\theta I_{i 5}(t)=0, \quad t_{1} \leq t \leq t_{3} . \\
\frac{d I_{i 6}(t)}{d t}=-B \lambda(t), \quad t_{4} \leq t \leq t_{5} . \\
\frac{d I_{i 7}(t)}{d t}=P(t)-\lambda(t), \quad t_{5} \leq t \leq T .
\end{gathered}
$$

With the boundary conditions $I_{i 1}(0)=0, I_{i 2}\left(t_{1}\right)=0, I_{i 3}\left(t_{3}\right)=0, I_{i 4}\left(t_{4}\right)=0, I_{i 5}\left(t_{1}\right)=W, I_{i 6}\left(t_{4}\right)=$ 0 , and $I_{i 7}(T)=0$, respectively, the above equations can be solved successively as follows: 


$$
\begin{aligned}
I_{i 1}(t)= & (\eta-1) \lambda_{0}\left[\frac{\Delta t^{2}}{2}+\frac{\delta \Delta t^{3}}{3}-\frac{(\theta-\Delta) \Delta t^{4}}{4}\right]-\lambda_{0}\left[t+\frac{\delta t^{2}}{2}-(\theta-\Delta) \frac{t^{3}}{2}\right], \\
I_{i 2}(t)= & (\eta-1) \lambda_{0}\left[\frac{\Delta}{2}\left(t^{2}-t_{1}^{2}\right)-\frac{\Delta \alpha t^{\beta}}{2}\left(t^{2}-t_{1}^{2}\right)+\frac{\delta \Delta}{3}\left(t^{3}-t_{1}^{3}\right)\right] \\
& -\lambda_{0}\left[\left(t-t_{1}\right)-\alpha t^{\beta}\left(t-t_{1}\right)+\frac{\delta}{2}\left(t^{2}-t_{1}^{2}\right)-\frac{\delta \alpha t^{\beta}}{2}\left(t^{2}-t_{1}^{2}\right)\right], \\
I_{i 3}(t)= & \lambda_{0}\left[\left(t_{3}-t\right)-\alpha t^{\beta}\left(t_{3}-t\right)+\frac{\delta}{2}\left(t_{3}^{2}-t^{2}\right)-\frac{\delta \alpha t^{\beta}}{2}\left(t_{3}^{2}-t^{2}\right)\right], \\
I_{i 4}(t)= & \lambda_{0}\left[\left(t_{4}-t\right)-\frac{\theta t^{2}}{2}\left(t_{4}-t\right)+\frac{\delta}{2}\left(t_{4}^{2}-t^{2}\right)-\frac{\delta \theta t^{2}}{4}\left(t_{4}^{2}-t^{2}\right)\right], \\
I_{i 5}(t)= & W e^{(-\theta / 2)\left(t_{1}^{2}-t^{2}\right)}, \\
I_{i 6}(t)= & \lambda_{0}\left[\left(t_{4}-t\right)+\left(\frac{\delta-s}{2}\right)\left(t_{4}^{2}-t^{2}\right)-\frac{\delta s}{3}\left(t_{4}^{3}-t^{3}\right)\right], \\
I_{7}(t)= & (\eta-1) \lambda_{0}\left[(t-T)+\frac{\Delta t^{2}}{2}(t-T)+\frac{\delta}{2}\left(t^{2}-T^{2}\right)+\frac{\delta \Delta t^{2}}{4}\left(t^{2}-T^{2}\right)\right] \\
& -\eta \lambda_{0}\left[(T-t)+\frac{\Delta t^{2}}{2}(T-t)+\frac{(\delta-\Delta)}{2}\left(T^{2}-t^{2}\right)\right] .
\end{aligned}
$$

Therefore, we find out some costs such as the following.

\subsection{Present Worth Ordering Cost}

Consider the following:

$$
\text { Ordering cost per cycle }=C_{1} e^{-r t_{5}} \text {. }
$$

\subsection{Present Worth Inventory Level in RW}

The total inventory level in RW can be derived as

$$
\begin{aligned}
I_{\mathrm{RW}}= & \int_{t_{1}}^{t_{2}} I_{2}(t) e^{-r t} d t+\int_{t_{2}}^{t_{3}} I_{3}(t) e^{-r t} d t \\
= & \lambda_{0}\left[(\eta-1)\left(\frac{\Delta}{2}\left(\frac{t_{2}^{3}}{3}+\frac{2 t_{1}^{3}}{3}\right)-\frac{\Delta r}{3}\left(\frac{t_{2}^{4}}{4}+\frac{t_{1}^{4}}{2}\right)+\frac{\Delta \delta}{3}\left(\frac{t_{2}^{4}}{4}+\frac{3 t_{1}^{4}}{4}\right)-\frac{r \Delta \delta}{3}\left(\frac{t_{2}^{5}}{5}+\frac{3 t_{1}^{5}}{10}\right)\right)\right] \\
& -\left[\left(\frac{t_{1}^{2}}{2}+\frac{t_{3}^{2}}{2}\right)-r\left(\frac{t_{1}^{3}}{6}+\frac{t_{3}^{3}}{6}\right)+\frac{\delta}{2}\left(\frac{2 t_{1}^{3}}{3}+\frac{2 t_{3}^{3}}{3}\right)-\frac{\delta r}{2}\left(\frac{t_{1}^{4}}{4}+\frac{t_{3}^{4}}{2}\right)\right] .
\end{aligned}
$$




\subsection{Present Worth Inventory Level in OW}

The total inventory level in OW can be derived as

$$
\begin{aligned}
I_{\mathrm{OW}}= & \int_{0}^{t_{1}} I_{1}(t) e^{-r t} d t+\int_{t_{1}}^{t_{3}} I_{5}(t) e^{-r t} d t+\int_{t_{3}}^{t_{4}} I_{4}(t) e^{-r t} d t \\
= & (\eta-1) \lambda_{0}\left[\frac{\Delta t_{1}^{3}}{6}-\frac{r \Delta t_{1}^{4}}{8}+\frac{\delta \Delta t_{1}^{4}}{12}\right]-\lambda_{0}\left[\frac{t_{1}^{2}}{2}-\frac{t_{4}^{2}}{2}-\frac{t_{3}^{2}}{2}-\frac{r t_{1}^{3}}{6}-\frac{r t_{3}^{3}}{3}-\frac{\delta t_{4}^{3}}{6}\right] \\
& +W\left[\left(t_{3}-t_{1}\right)-\frac{r}{2}\left(t_{3}^{2}-t_{1}^{2}\right)+\frac{\theta t_{1}^{3}}{2}\right] .
\end{aligned}
$$

\subsection{Present Worth Deteriorated Items}

The total quantity of deteriorated items during the period $(0, T)$ is given by

$$
\begin{aligned}
I_{D}= & \int_{0}^{t_{1}} \theta t I_{i 1}(t) e^{-r t} d t+\int_{t_{1}}^{t_{3}} \theta t I_{i 5}(t) e^{-r t} d t \\
& +\int_{t_{3}}^{t_{4}} \theta t I_{i 4}(t) e^{-r t} d t+\int_{t_{1}}^{t_{2}} \alpha \beta t^{\beta-1} I_{i 2}(t) e^{-r t} d t+\int_{\mathrm{t}_{2}}^{t_{3}} \alpha \beta t^{\beta-1} I_{i 3}(t) e^{-r t} d t \\
= & \theta \theta \lambda_{0}\left\{(\eta-1)\left(\frac{\Delta t_{1}^{4}}{8}+\frac{\Delta \delta t_{1}^{5}}{15}+\frac{\Delta r t_{1}^{5}}{10}\right)-\left(\frac{t_{1}^{3}}{3}+\frac{\delta t_{1}^{4}}{8}+\frac{r t_{1}^{4}}{4}\right)\right\} \\
& +W \theta\left\{\frac{t_{3}^{2}}{2}-\frac{t_{1}^{2}}{2}+\frac{\theta t_{3}^{4}}{8}-\frac{\theta t_{1}^{4}}{8}-\frac{r t_{3}^{3}}{3}+\frac{r t_{1}^{3}}{3}\right\} \\
& +\theta \lambda_{0}\left\{\left(-\frac{t_{3}^{3}}{6}-\frac{t_{4}^{3}}{3}\right)-r\left(-\frac{t_{3}^{4}}{12}-\frac{t_{4}^{4}}{4}\right)+\frac{\delta r}{2}\left(-\frac{2 t_{3}^{5}}{15}-\frac{t_{4}^{5}}{5}\right)\right\} \\
& +\lambda_{0} \alpha \beta\left\{\left\{(\eta-1) \Delta\left(\frac{t_{2}^{\beta+2}}{2(\beta+2)}+\frac{t_{1}^{\beta+2}}{2 \beta(\beta+2)}\right)\right\}-\left(\frac{t_{2}^{\beta+1}}{(\beta+1)}+\frac{t_{1}^{\beta+1}}{\beta(\beta+1)}\right)\right\} \\
& \left.\times \lambda_{0} \alpha \beta\left\{\left(\frac{t_{2}^{\beta+1}}{(\beta+1)}+\frac{t_{3}^{\beta+1}}{\beta(\beta+1)}\right)-r\left(\frac{t_{2}^{\beta+2}}{(\beta+2)}+\frac{t_{3}^{\beta+2}}{(\beta+2)(\beta+1)}\right)\right\}\right]
\end{aligned}
$$




\subsection{Present Worth Shortage Cost}

Total quantity of shortage units $\left(I_{S}\right)$ during the period $(0, T)$ is given by

$$
\begin{aligned}
I_{S}= & -\int_{t_{4}}^{t_{5}} I_{i 6}(t) e^{-r t} d t-\int_{t_{5}}^{T} I_{i 7}(t) e^{-r t} d t \\
= & s\left[\lambda_{0}\left\{\left(\frac{t_{4}^{2}}{2}+\frac{t_{5}^{2}}{2}\right)-r\left(\frac{t_{4}^{3}}{6}+\frac{t_{5}^{3}}{3}\right)-\frac{\delta s}{3}\left(\frac{3 t_{4}^{4}}{4}+\frac{t_{5}^{4}}{4}\right)\right\}\right. \\
& \left.+(\eta-1) \lambda_{0}\left\{\left(\frac{T^{2}}{2}+\frac{t_{5}^{2}}{2}\right)-r\left(\frac{T^{3}}{6}+\frac{t_{5}^{3}}{3}\right)\right\}+\eta \lambda_{0}\left\{\left(\frac{T^{2}}{2}+\frac{t_{5}^{2}}{2}\right)-r\left(\frac{T^{3}}{6}+\frac{t_{5}^{3}}{3}\right)\right\}\right] .
\end{aligned}
$$

\subsection{Present Worth Lost Sales Quantity}

The total amount of lost sales $\left(I_{L}\right)$ during the period $(0, T)$ can be obtained as

$$
I_{L}=\int_{t_{4}}^{t_{5}}\left\{1-e^{-s t}\right\} \lambda_{0} e^{\delta t} d t=s \lambda_{0}\left[\frac{1}{2}\left(t_{5}^{2}-t_{4}^{2}\right)+\frac{\delta}{3}\left(t_{5}^{3}-t_{4}^{3}\right)\right]
$$

Number of defective items is

$$
N=\left\{\begin{array}{cc}
0, & X \geq t_{2} \\
\int_{X}^{t_{2}} P d t, & X<t_{2}
\end{array}\right\}, \quad N=t_{2}\left\{\begin{array}{cc}
0, & X \geq t_{2} \\
P\left(t_{2}-X\right), & X<t_{2}
\end{array}\right\} .
$$

Expected number of defective items in a production cycle is

$$
E(N)=\int_{0}^{t_{2}} P\left(t_{2}-X\right) f(X) d X
$$

$f(X)$ is probability density function of $X$ :

$$
f(X)=\mu e^{-\mu X} .
$$




\subsection{Present Worth of Rework Cost}

The total amount of rework cost $\left(I_{R}\right)$ during the period $\left(0, T_{2}\right)$ can be obtained as

$$
\begin{aligned}
I_{R}=E(N) e^{-r t_{2}} & \left\{\int_{0}^{t_{2}} P\left(t_{2}-X\right) f(X) d X\right\} e^{-r t_{2}} \\
=\lambda_{0} \mu & {\left[(\eta-1) \Delta\left\{\frac{\Delta}{8}\left(\frac{\mu t_{2}^{6}}{6}-\frac{t_{2}^{5}}{5}\right)+\frac{\Delta \delta}{15}\left(\frac{\mu t_{2}^{7}}{7}-\frac{t_{2}^{6}}{6}\right)\right\}-\Delta\left\{\frac{1}{3}\left(\frac{\mu t_{2}^{5}}{5}-\frac{t_{2}^{4}}{4}\right)+\frac{\delta}{8}\left(\frac{\mu t_{2}^{6}}{6}-\frac{t_{2}^{5}}{5}\right)\right\}\right.} \\
& +\eta \Delta\left\{\frac{1}{2}\left(\frac{\mu t_{2}^{4}}{4}-\frac{t_{2}^{3}}{3}\right)+\frac{\delta}{6}\left(\frac{\mu t_{2}^{5}}{5}-\frac{t_{2}^{4}}{4}\right)\right\} \\
& +(\eta-1) \Delta\left\{\frac{\Delta}{2}\left(\frac{t_{2}^{5}}{4}-\frac{\mu t_{2}^{6}}{8}\right)-\frac{\Delta^{2} \alpha}{2}\left(\frac{t_{2}^{\beta+5}}{\beta+4}-\frac{\mu t_{2}^{\beta+6}}{2(\beta+4)}\right)\right\} \\
& \left.-\left\{\left(\frac{t_{2}^{4}}{3}-\frac{\mu t_{2}^{5}}{6}\right)-\alpha\left(\frac{t_{2}^{\beta+4}}{\beta+3}-\frac{\mu t_{2}^{\beta+5}}{2(\beta+3)}\right)\right\}+\eta \Delta\left(\frac{t_{2}^{3}}{2}+\frac{\delta t_{2}^{4}}{3}-\frac{\mu t_{2}^{4}}{4}\right)\right]\left(1-r t_{2}\right) .
\end{aligned}
$$

\subsection{Present Worth of Total Cost}

The total average cost (TC) of the inventory system is given by

$$
\mathrm{TC}=\frac{1}{T}\left[C_{1}+C_{\mathrm{RW}} I_{\mathrm{RW}}+C_{\mathrm{OW}} I_{\mathrm{OW}}+C_{2} I_{D}+C_{3} I_{S}+C_{4} I_{L}+C_{5} I_{R}\right]
$$

\section{Fuzzy Model}

In order to develop the model in a fuzzy environment, we consider the costs as the triangular fuzzy numbers where $\tilde{C}_{1}=\left(C_{1}-\Delta_{1}, C_{1}, C_{1}+\Delta_{2}\right), \tilde{C}_{\mathrm{RW}}=\left(C_{\mathrm{RW}}-\Delta_{3}, C_{\mathrm{RW}}, C_{\mathrm{RW}}+\Delta_{4}\right), \tilde{C}_{\mathrm{OW}}=$ $\left(C_{\text {OW }}-\Delta_{5}, C_{\text {OW }}, C_{\text {OW }}+\Delta_{6}\right), \widetilde{C}_{2}=\left(C_{2}-\Delta_{7}, C_{2}, C_{2}+\Delta_{8}\right), \widetilde{C}_{3}=\left(C_{3}-\Delta_{9}, C_{3}, C_{3}+\Delta_{10}\right), \widetilde{C}_{4}=$ $\left(C_{4}-\Delta_{11}, C_{4}, C_{4}+\Delta_{12}\right)$, and $\widetilde{C}_{5}=\left(C_{5}-\Delta_{13}, C_{5}, C_{5}+\Delta_{14}\right)$ such that $0<\Delta_{1}<C_{1}, 0<\Delta_{2}$, $0<\Delta_{3}<C_{\mathrm{RW}}, 0<\Delta_{4}, 0<\Delta_{5}<\mathrm{C}_{\mathrm{OW}}, 0<\Delta_{6}, 0<\Delta_{7}<C_{2}, 0<\Delta_{8}, 0<\Delta_{9}<C_{3}, 0<\Delta_{10}$, $0<\Delta_{11}<C_{4}, 0<\Delta_{12}, 0<\Delta_{13}<C_{5}, 0<\Delta_{14}$, and $\Delta_{1}, \Delta_{2}, \Delta_{3}, \Delta_{4}, \Delta_{5}, \Delta_{6}, \Delta_{7}, \Delta_{8}, \Delta_{9}, \Delta_{10}$, $\Delta_{11}, \Delta_{12}, \Delta_{13}$, and $\Delta_{14}$ are determined by the decision maker based on the uncertainty of the problem. Thus, the costs are considered as the fuzzy numbers with membership function: 


$$
\begin{aligned}
& \mathrm{TC}=\frac{1}{T}\left[\widetilde{C}_{1}+\widetilde{C}_{\mathrm{RW}} I_{\mathrm{RW}}+\widetilde{C}_{\mathrm{OW}} I_{\mathrm{OW}}+\widetilde{C}_{2} I_{D}+\widetilde{C}_{3} I_{S}+\widetilde{C}_{4} I_{L}+\widetilde{C}_{5} I_{R}\right] \\
& \widetilde{C}_{1}=\left(C_{1}-\Delta_{1}, C_{1}, C_{1}+\Delta_{2}\right), \\
& \widetilde{C}_{\mathrm{RW}}=\left(C_{\mathrm{RW}}-\Delta_{3}, C_{\mathrm{RW}}, C_{\mathrm{RW}}+\Delta_{4}\right) \text {, } \\
& \widetilde{C}_{\mathrm{OW}}=\left(C_{\mathrm{OW}}-\Delta_{5}, C_{\mathrm{OW}}, C_{\mathrm{OW}}+\Delta_{6}\right), \\
& \widetilde{C}_{2}=\left(C_{2}-\Delta_{7}, C_{2}, C_{2}+\Delta_{8}\right) \text {, } \\
& \widetilde{C}_{3}=\left(C_{3}-\Delta_{9}, C_{3}, C_{3}+\Delta_{10}\right), \\
& \widetilde{C}_{4}=\left(C_{4}-\Delta_{11}, C_{4}, C_{4}+\Delta_{12}\right), \\
& \widetilde{C}_{5}=\left(C_{5}-\Delta_{13}, C_{5}, C_{5}+\Delta_{14}\right) \text {. }
\end{aligned}
$$

By Centroid Method consider

$$
\begin{aligned}
& \tilde{C}_{1}=C_{1}+\frac{1}{3}\left(\Delta_{2}-\Delta_{1}\right) \\
& \widetilde{C}_{\mathrm{RW}}=C_{\mathrm{RW}}+\frac{1}{3}\left(\Delta_{4}-\Delta_{3}\right) \\
& \tilde{C}_{\mathrm{OW}}=C_{\mathrm{OW}}+\frac{1}{3}\left(\Delta_{6}-\Delta_{5}\right) \\
& \tilde{C}_{2}=C_{2}+\frac{1}{3}\left(\Delta_{8}-\Delta_{7}\right) \\
& \tilde{C}_{3}=C_{3}+\frac{1}{3}\left(\Delta_{10}-\Delta_{9}\right) \\
& \widetilde{C}_{4}=C_{4}+\frac{1}{3}\left(\Delta_{12}-\Delta_{11}\right) \\
& \tilde{C}_{5}=C_{5}+\frac{1}{3}\left(\Delta_{14}-\Delta_{13}\right), \\
& F_{1}=\frac{1}{T}\left[\left(C_{1}+\Delta_{2}\right)+\left(C_{\mathrm{RW}}+\Delta_{4}\right) I_{\mathrm{RW}}\right. \\
& \left.+\left(C_{\mathrm{OW}}+\Delta_{6}\right) I_{\mathrm{OW}}+\left(C_{2}+\Delta_{8}\right) I_{D}+\left(C_{3}+\Delta_{10}\right) I_{S}+\left(C_{4}+\Delta_{12}\right) I_{L}+\left(C_{5}+\Delta_{14}\right) I_{R}\right] \\
& F_{2}=\frac{1}{T}\left[C_{1}+C_{\mathrm{RW}} I_{\mathrm{RW}}+C_{\mathrm{OW}} I_{\mathrm{OW}}+C_{2} I_{D}+C_{3} I_{S}+C_{4} I_{L}+C_{5} I_{R}\right], \\
& F_{3}=\frac{1}{T}\left[\left(C_{1}-\Delta_{1}\right)+\left(C_{\mathrm{RW}}-\Delta_{3}\right) I_{\mathrm{RW}}\right. \\
& \left.+\left(C_{\mathrm{OW}}-\Delta_{5}\right) I_{\mathrm{OW}}+\left(C_{2}-\Delta_{7}\right) I_{D}+\left(C_{3}-\Delta_{9}\right) I_{S}+\left(C_{4}-\Delta_{11}\right) I_{L}+\left(C_{5}-\Delta_{13}\right) I_{R}\right] .
\end{aligned}
$$


By defuzzification, we get

$$
\begin{aligned}
=\frac{1}{T}[ & C_{1}+\frac{1}{4}\left(\Delta_{2}-\Delta_{1}\right)+C_{\mathrm{RW}} I_{\mathrm{RW}}+\frac{1}{4}\left(\Delta_{4}-\Delta_{3}\right)+C_{\mathrm{OW}} I_{\mathrm{OW}}+\frac{1}{4}\left(\Delta_{6}-\Delta_{5}\right)+C_{2} I_{D} \\
& \left.+\frac{1}{4}\left(\Delta_{8}-\Delta_{7}\right)+C_{3} I_{S}+\frac{1}{4}\left(\Delta_{10}-\Delta_{9}\right)+C_{4} I_{L}+\frac{1}{4}\left(\Delta_{12}-\Delta_{11}\right)+C_{5} I_{R}+\frac{1}{4}\left(\Delta_{14}-\Delta_{13}\right)\right] .
\end{aligned}
$$

\section{Numerical Example}

To illustrate the theory of the model we consider the following data on the basis of the previous study.

$$
\begin{aligned}
& \delta=2, \quad \mathrm{C}_{\mathrm{RW}}=0.6, \quad W=50, \quad \theta=0.03, \\
& \mathrm{C}_{1}=10, \quad \mathrm{C}_{\mathrm{OW}}=1.2, \quad \eta=5, \quad \mathrm{C}_{2}=2, \\
& \alpha=0.06, \quad \beta=0.01, \quad C_{3}=5, \quad \mu=0.005 \text {, } \\
& \mathrm{C}_{4}=6, \quad \mathrm{C}_{5}=0.1, \quad \lambda_{0}=20, \quad T=10
\end{aligned}
$$

in appropriate units. Based on these input data, the findings are as follows So the optimum values of

$$
\begin{array}{lll}
t_{1}^{*}=1.42655, & t_{2}^{*}=2.08592, & t_{3}^{*}=4.17695, \\
t_{4}^{*}=5.72968, & t_{5}^{*}=8.38866, & \mathrm{TC}=4007.4 .
\end{array}
$$

\section{Fuzzy Numerical}

$$
\begin{aligned}
& \tilde{C}_{1}=(9,10,11), \quad \tilde{C}_{\mathrm{OW}}=(1.1,1.2,1.3), \quad \eta=5, \quad \lambda_{0}=20, \\
& \delta=2, \quad \tilde{C}_{\mathrm{RW}}=(0.5,0.6,0.7), \quad W=50, \quad \theta=0.03, \\
& \tilde{C}_{2}=(1.5,2,2.5), \quad \alpha=0.06, \quad \beta=0.01, \quad \tilde{C}_{3}=(4,5,6), \\
& \mu=0.005, \quad \tilde{C}_{4}=(5.5,6,6.5), \quad \tilde{C}_{5}=(0.09,0.1,0.2), \quad T=10 \\
& t_{1}^{*}=(1.41419,1.42655,1.44654), \quad t_{2}^{*}=(2.08595,2.08592,2.06724), \\
& t_{3}^{*}=(4.16689,4.17695,4.23937), \quad t_{4}^{*}=(5.84037,5.72968,5.69694), \\
& t_{5}^{*}=(8.90087,8.38666,8.08675), \quad \mathrm{TC}=(3425.62,4007.4,4628.42) .
\end{aligned}
$$

\section{Sensitivity Analysis}

See Table 1. 
Table 1

\begin{tabular}{|c|c|c|c|c|c|c|c|}
\hline Parameter & $\begin{array}{l}\text { Change in } \\
\text { parameter }\end{array}$ & $t_{1}^{*}$ & $t_{2}^{*}$ & $t_{3}^{*}$ & $t_{4}^{*}$ & $t_{5}^{*}$ & $\mathrm{TC}$ \\
\hline \multirow{5}{*}{$C_{1}$} & 12 & 1.42651 & 2.08592 & 4.17679 & 5.72944 & 8.38843 & 4007.35 \\
\hline & 14 & 1.42648 & 2.08592 & 4.17664 & 5.7292 & 8.3882 & 4007.3 \\
\hline & 16 & 1.42644 & 2.08592 & 4.17648 & 5.72897 & 8.38797 & 4007.25 \\
\hline & 18 & 1.4264 & 2.08592 & 4.17633 & 5.72873 & 8.38774 & 4007.2 \\
\hline & 20 & 1.42637 & 2.08592 & 4.17617 & 5.7285 & 8.3875 & 4007.15 \\
\hline \multirow{5}{*}{$\alpha$} & 0.07 & 1.42891 & 2.08533 & 4.17599 & 5.72978 & 8.38871 & 4007.42 \\
\hline & 0.08 & 1.43164 & 2.08474 & 4.17505 & 5.72988 & 8.38877 & 4007.43 \\
\hline & 0.09 & 1.43472 & 2.08414 & 4.17413 & 5.72998 & 8.38833 & 4007.44 \\
\hline & 0.1 & 1.43816 & 2.08355 & 4.17321 & 5.73009 & 8.38889 & 4007.45 \\
\hline & 0.2 & 1.49168 & 2.07793 & 4.16471 & 5.73122 & 8.38951 & 4007.46 \\
\hline \multirow{5}{*}{$\beta$} & 0.02 & 1.428 & 2.08244 & 4.17683 & 5.72969 & 8.38866 & 4007.41 \\
\hline & 0.03 & 1.42945 & 2.07897 & 4.17672 & 5.7297 & 8.38867 & 4007.42 \\
\hline & 0.04 & 1.4309 & 2.07552 & 4.17662 & 5.72971 & 8.38867 & 4007.43 \\
\hline & 0.05 & 1.43236 & 2.07209 & 4.1765 & 5.72972 & 8.38868 & 4007.44 \\
\hline & 0.06 & 1.43381 & 2.06868 & 4.17639 & 5.72973 & 8.38869 & 4007.45 \\
\hline \multirow{5}{*}{$\lambda_{0}$} & 22 & 1.34214 & 2.08549 & 4.17764 & 5.72985 & 8.3888 & 4407.61 \\
\hline & 24 & 1.27294 & 2.08518 & 4.1764 & 5.7298 & 8.38881 & 4807.68 \\
\hline & 26 & 1.21496 & 2.08495 & 4.1742 & 5.72965 & 8.38876 & 5207.94 \\
\hline & 28 & 1.16551 & 2.08476 & 4.17155 & 5.72945 & 8.38868 & 5608.08 \\
\hline & 30 & 1.12271 & 2.08462 & 4.16873 & 5.72922 & 8.38857 & 6008.2 \\
\hline \multirow{5}{*}{$\delta$} & 2.1 & 1.44449 & 2.08501 & 4.22808 & 5.81669 & 8.46614 & 4048.1 \\
\hline & 2.2 & 1.46366 & 2.08411 & 4.27917 & 5.90235 & 8.5434 & 4089.6 \\
\hline & 2.3 & 1.48418 & 2.08325 & 4.33018 & 5.98681 & 8.62043 & 4131.9 \\
\hline & 2.4 & 1.50622 & 2.08242 & 4.38107 & 6.07019 & 8.69721 & 4175 \\
\hline & 2.5 & 1.52996 & 2.08161 & 4.43178 & 6.15258 & 8.77374 & 4218.9 \\
\hline \multirow{5}{*}{$\Delta$} & 0.32 & 1.34242 & 1.91621 & 4.18507 & 5.73049 & 8.38911 & 4007.8 \\
\hline & 0.34 & 1.27138 & 1.7726 & 4.19123 & 5.73111 & 8.38945 & 4008.13 \\
\hline & 0.36 & 1.2103 & 1.65176 & 4.19603 & 5.7316 & 8.38972 & 4008.4 \\
\hline & 0.38 & 1.15704 & 1.54628 & 4.19987 & 5.73199 & 8.38994 & 4008.63 \\
\hline & 0.4 & 1.11006 & 1.45395 & 4.20299 & 5.7323 & 8.39011 & 4008.83 \\
\hline \multirow{5}{*}{ Cow } & 1.4 & 1.24759 & 2.08895 & 4.08125 & 5.72016 & 8.3834 & 4004.78 \\
\hline & 1.6 & 1.12142 & 2.09152 & 3.99301 & 5.70965 & 8.37761 & 4002.17 \\
\hline & 1.8 & 1.02623 & 2.09365 & 3.85523 & 5.69852 & 8.37149 & 3999.63 \\
\hline & 2.0 & 0.951127 & 2.09543 & 3.72869 & 5.68693 & 8.36512 & 3997.18 \\
\hline & 2.2 & 0.889949 & 2.09691 & 3.59326 & 5.67493 & 8.35856 & 3994.86 \\
\hline \multirow{5}{*}{$C_{\mathrm{RW}}$} & 0.7 & 1.56693 & 2.08682 & 4.31716 & 5.75917 & 8.40501 & 4013.99 \\
\hline & 0.8 & 1.70794 & 2.08788 & 4.4499 & 5.78821 & 8.42119 & 4020.78 \\
\hline & 0.9 & 1.85215 & 2.08912 & 4.57497 & 5.81672 & 8.43716 & 4027.78 \\
\hline & 1.0 & 2.00219 & 2.09058 & 4.69176 & 5.84457 & 8.45284 & 4034.98 \\
\hline & 1.1 & 2.16108 & 2.0923 & 4.79913 & 5.87156 & 8.46812 & 4042.38 \\
\hline \multirow{5}{*}{$C_{3}$} & 5.2 & 1.42214 & 2.08589 & 4.15801 & 5.70089 & 8.25883 & 4101 \\
\hline & 5.4 & 1.4188 & 2.08586 & 4.14345 & 5.67877 & 8.14299 & 4198.27 \\
\hline & 5.6 & 1.41632 & 2.08584 & 4.13251 & 5.66213 & 8.03918 & 4298.68 \\
\hline & 5.8 & 1.41454 & 2.08583 & 4.12454 & 5.65002 & 7.94579 & 4401.77 \\
\hline & 6.0 & 1.41331 & 2.08582 & 4.11904 & 5.64165 & 7.86147 & 4507.17 \\
\hline
\end{tabular}


Table 1: Continued.

\begin{tabular}{|c|c|c|c|c|c|c|c|}
\hline Parameter & $\begin{array}{l}\text { Change in } \\
\text { parameter }\end{array}$ & $t_{1}^{*}$ & $t_{2}^{*}$ & $t_{3}^{*}$ & $t_{4}^{*}$ & $t_{5}^{*}$ & $\mathrm{TC}$ \\
\hline \multirow{5}{*}{$C_{4}$} & 6.1 & 1.42907 & 2.08594 & 4.18761 & 5.74588 & 8.44763 & 4036.48 \\
\hline & 6.2 & 1.43177 & 2.08596 & 4.19892 & 5.76306 & 8.50755 & 4066.33 \\
\hline & 6.3 & 1.43467 & 2.08598 & 4.21088 & 5.78123 & 8.5684 & 4096.98 \\
\hline & 6.4 & 1.43776 & 2.08601 & 4.22349 & 5.8004 & 8.63019 & 4128.45 \\
\hline & 6.5 & 1.44106 & 2.08603 & 4.23676 & 5.82055 & 8.69293 & 4160.76 \\
\hline \multirow{5}{*}{$C_{5}$} & 0.12 & 1.42648 & 2.08134 & 4.17695 & 5.72968 & 8.38866 & 4007.41 \\
\hline & 0.14 & 1.42641 & 2.07681 & 4.17696 & 5.72968 & 8.38866 & 4007.41 \\
\hline & 0.16 & 1.42634 & 2.07232 & 4.17697 & 5.72968 & 8.38866 & 4007.42 \\
\hline & 0.18 & 1.42627 & 2.06786 & 4.17698 & 5.72968 & 8.38866 & 4007.42 \\
\hline & 0.2 & 1.4262 & 2.06345 & 4.17699 & 5.72968 & 8.38866 & 4007.43 \\
\hline \multirow{5}{*}{$r$} & 0.16 & 1.3601 & 2.13665 & 3.84454 & 5.20265 & 7.86865 & 3662.23 \\
\hline & 0.17 & 1.31431 & 2.19421 & 3.55049 & 4.72879 & 7.40461 & 3348.43 \\
\hline & 0.18 & 1.28124 & 2.26059 & 3.29027 & 4.29964 & 6.98831 & 3059.69 \\
\hline & 0.19 & 1.25646 & 2.33895 & 3.0598 & 3.90794 & 6.61292 & 2791.16 \\
\hline & 0.2 & 1.23733 & 2.4348 & 2.85541 & 3.54734 & 6.27281 & 2539.13 \\
\hline \multirow{5}{*}{$s$} & 0.6 & 1.51085 & 2.08657 & 4.48099 & 6.1919 & 8.39432 & 4033.45 \\
\hline & 0.7 & 1.5898 & 2.08718 & 4.6929 & 6.51616 & 8.39547 & 4051.61 \\
\hline & 0.8 & 1.66348 & 2.08777 & 4.8477 & 6.75601 & 8.39463 & 4064.91 \\
\hline & 0.9 & 1.73232 & 2.08835 & 4.96477 & 6.94047 & 8.3929 & 4075.05 \\
\hline & 1.0 & 1.7968 & 2.08891 & 5.05568 & 7.08662 & 8.39076 & 4083.01 \\
\hline \multirow{5}{*}{$W$} & 55 & 1.52163 & 2.08646 & 4.1731 & 5.72929 & 8.38844 & 4007.93 \\
\hline & 60 & 1.61945 & 2.08709 & 4.16542 & 5.72852 & 8.38802 & 4008.42 \\
\hline & 65 & 1.72024 & 2.08781 & 4.15332 & 5.72731 & 8.38735 & 4008.87 \\
\hline & 70 & 1.82396 & 2.08864 & 4.13619 & 5.7256 & 8.3864 & 4009.26 \\
\hline & 75 & 1.9302 & 2.08957 & 4.11348 & 5.72336 & 8.38516 & 4009.62 \\
\hline \multirow{5}{*}{$\eta$} & 6 & 1.14672 & 1.5716 & 4.00831 & 5.44543 & 8.11066 & 4627.9 \\
\hline & 7 & 0.988027 & 1.26651 & 3.87949 & 5.23885 & 7.90997 & 5256.31 \\
\hline & 8 & 0.881603 & 1.06271 & 3.7803 & 5.08204 & 7.75838 & 5889.69 \\
\hline & 9 & 0.803577 & 0.916472 & 3.70204 & 4.95889 & 7.63982 & 6526.39 \\
\hline & 10 & 0.743094 & 0.8063 & 3.63886 & 4.85959 & 7.54456 & 7165.4 \\
\hline
\end{tabular}

\section{Observations}

(1) As we increase the ordering cost, the optimum value of $t_{1}^{*}, t_{3}^{*}, t_{4}^{*}, t_{5}^{*}$ decreases, and $t_{2}^{*}$ remains same. By this effect, our total cost decreases.

(2) As we increase the deterioration parameter for OW, the optimum value of $t_{1}^{*}, t_{2}^{*}$ increases and that of $t_{3}^{*}, t_{4}^{*}, t_{5}^{*}$ decreases, so our total cost decreases.

(3) As we increase the deterioration parameters for RW, the optimum value of $t_{1}^{*}, t_{4}^{*}, t_{5}^{*}$, increases and that of $t_{2}^{*}, t_{3}^{*}$ decreases then total cost slightly increase decreases. So the total cost is minimum when the deterioration rate of own warehouse is less than the deterioration rate of rented warehouse. 


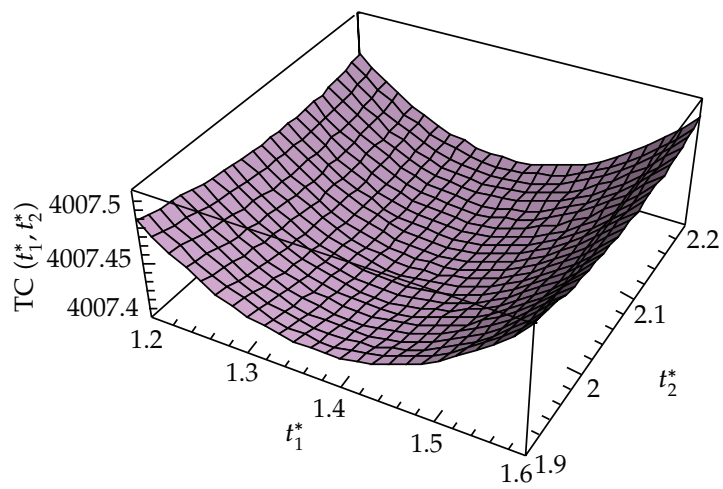

Figure 2: Convexity of total cost with respect to $t_{1}^{*}$ and $t_{2}^{*}$.

(4) As we increase the demand parameter $\lambda_{0}$, the optimum value of $t_{1}^{*}, t_{2}^{*}, t_{3}^{*}, t_{4}^{*}, t_{5}^{*}$ decreases, while total cost increases. More demand encourages more production which increases the total cost.

(5) As we increase the demand parameter $\delta$, the optimum value of $t_{1}^{*}, t_{3}^{*}, t_{4}^{*}, t_{5}^{*}$ increases and $t_{2}^{*}$ decreases, and then our total cost increases. In both the cases, we see that as we increase the demand, increase in total cost is then obvious.

(6) On increasing the imperfect rate, the optimum value of $t_{1}^{*}, t_{3}^{*}, t_{4}^{*}, t_{5}^{*}$ increases and $t_{2}^{*}$ decreases; then our total cost slightly increases. This is very obvious since with an increment in the percentage of the defective items the total cost then increases.

(7) As we increase the holding cost for OW, the optimum value of $t_{1}^{*}, t_{3}^{*}, t_{4}^{*}$, $t_{5}^{*}$ decreases and $t_{2}^{*}$ increases, and then our total cost decreases.

(8) As we increase the holding cost for RW, the optimum value of $t_{1}^{*}, t_{2}^{*}, t_{3}^{*}, t_{4}^{*}, t_{5}^{*}$ increases then our total cost increases.

(9) On increasing the shortage cost, the optimum value of $t_{1}^{*}, t_{2}^{*}, t_{3}^{*}, t_{4}^{*}, t_{5}^{*}$ decreases, and then our total cost increases.

(10) As we increase the lost sale cost, the optimum value of $t_{1}^{*}, t_{2}^{*}, t_{3}^{*}, t_{4}^{*}, t_{5}^{*}$ increases, and then our total cost increases.

(11) As we increase the rework cost, the optimum value of $t_{1}^{*}, t_{2}^{*}$ decreases, $t_{3}^{*}$ increases, and $t_{4}^{*}, t_{5}^{*}$ remains the same, and so our total cost slightly increases.

(12) As we increase the inflation rate the optimum value of $t_{1}^{*}, t_{3}^{*}, t_{4}^{*}, t_{5}^{*}$ decreases and $t_{2}^{*}$ increases, while total cost decreases.

(13) As we increase the backlogging parameter, the optimum value of $t_{1}^{*}, t_{2}^{*}, t_{3}^{*}, t_{4}^{*}, t_{5}^{*}$ and total cost increase.

(14) As we increase the warehouse capacity, the optimum value of $t_{1}^{*}, t_{2}^{*}$ increases and $t_{3}^{*}, t_{4}^{*}, t_{5}^{*}$ decreases, and so our total cost increases. As we increase the warehouse capacity, our expenses increases, and so total cost also increases. 


\section{Conclusion}

This paper presented a warehouse imperfect production-inventory models for deteriorating items having time varying demand patterns with Weibull distribution deterioration and partial backlogging under the effect of inflation and time value of money. Inflation plays a very significant role in the inventory models. Demand, production, defective item production, and so forth are natural in any manufacturing system. In this paper, we have developed two models for a manufacturing system. In the first model, all costs were assumed to be stochastic whereas in the second model, the costs were assumed to be fuzzy. The production rate was proportional to the demand rate. Well-known triangular membership function was used for all the fuzzy numbers. Numerical examples are solved by the software MATHEMATICA 8.0. Convexity of the total average cost is also illustrated in Figure 2. However, the advantage of the fuzzy approach is that it relaxes the assumptions such as defective rate, demand rate etc. Also, it eases the difficulties in searching for suitable probability distribution function to represent the random behavior of uncontrollable variables.

\section{References}

[1] M. J. Rosenblatt and H. L. Lee, "Economic production cycles with imperfect production processes," IIE Transactions, vol. 18, no. 1, pp. 48-55, 1986.

[2] C. H. Kim and Y. Hong, "An optimal production run length in deteriorating production processes," International Journal of Production Economics, vol. 58, no. 2, pp. 183-189, 1999.

[3] K. J. Chung and K. L. Hou, "An optimal production run time with imperfect production processes and allowable shortages," Computers and Operations Research, vol. 30, no. 4, pp. 483-490, 2003.

[4] C. Singh and S. R. Singh, "Imperfect production process with exponential demand rate, Weibull deterioration under inflation," International Journal of Operational Research, vol. 12, no. 4, pp. 430-445, 2011.

[5] L. A. Zadeh, "Fuzzy sets," Information and Control, vol. 8, no. 3, pp. 338-353, 1965.

[6] R. E. Bellman and L. A. Zadeh, "Decision-making in a fuzzy environment," Management Science, vol. 17, no. 4, pp. B141-B164, 1970.

[7] M. Gen, Y. Tsujimura, and D. Zheng, "An application of fuzzy set theory to inventory control models," Computers and Industrial Engineering, vol. 33, no. 3-4, pp. 553-556, 1997.

[8] J. S. Yao and J. Chiang, "Inventory without backorder with fuzzy total cost and fuzzy storing cost defuzzified by centroid and signed distance," European Journal of Operational Research, vol. 148, no. 2, pp. 401-409, 2003.

[9] S. Mondal and M. Maiti, "Multi-item fuzzy EOQ models using genetic algorithm," Computers and Industrial Engineering, vol. 44, no. 1, pp. 105-117, 2003.

[10] M. K. Maiti and M. Maiti, "Fuzzy inventory model with two warehouses under possibility constraints," Fuzzy Sets and Systems, vol. 157, no. 1, pp. 52-73, 2006.

[11] N. K. Mahapatra and M. Maiti, "A fuzzy stochastic approach to multi-objective inventory model of deteriorating items with various types of demand and time dependent holding cost," Journal of the Operational Research Society of India, vol. 43, no. 2, pp. 117-131, 2006.

[12] K. A. Halim, B. C. Giri, and K. S. Chaudhuri, "Fuzzy economic order quantity model for perishable items with stochastic demand, partial backlogging and fuzzy deterioration rate," International Journal of Operational Research, vol. 3, no. 1-2, pp. 77-96, 2008.

[13] A. N. Gani and S. Maheswari, "Supply chain model for the retailer's ordering policy under two levels of delay payments in fuzzy environment," Applied Mathematical Sciences, vol. 4, no. 21-24, pp. 11551164, 2010.

[14] H. M. Lee and J. S. Yao, "Economic production quantity for fuzzy demand quantity and fuzzy production quantity," European Journal of Operational Research, vol. 109, no. 1, pp. 203-211, 1998.

[15] K. A. Halim, B. C. Giri, and K. S. Chaudhuri, "Lot sizing in an unreliable manufacturing system with fuzzy demand and repair time," International Journal of Industrial and Systems Engineering, vol. 5, no. 4, pp. 485-500, 2010. 
[16] S. H. Chen and S. M. Chang, "Optimization of fuzzy production inventory model with unrepairable defective products," International Journal of Production Economics, vol. 113, no. 2, pp. 887-894, 2008.

[17] J. A. Buzacott, "Economic order quantities with inflation," Operational Research Quarterly, vol. 26, no. 3, pp. 553-558, 1975.

[18] H. Bierman and J. Thomas, "Inventory decisions under inflationary conditions," Decision Sciences, vol. 8, no. 1, pp. 151-155, 1977.

[19] R. B. Misra, “A study of inflation effects on inventory system," Logistics Spectrum, vol. 9, pp. 260-268, 1979.

[20] M. J. Chandra and M. L. Bahner, "The effects of inflation and time value of money on some inventory systems," International Journal of Production Research, vol. 23, no. 4, pp. 723-730, 1985.

[21] I. Moon and S. Lee, "Effects of inflation and time-value of money on an economic order quantity model with a random product life cycle," European Journal of Operational Research, vol. 125, no. 3, pp. 588-601, 2000.

[22] S. T. Lo, H. M. Wee, and W. C. Huang, "An integrated production-inventory model with imperfect production processes and Weibull distribution deterioration under inflation," International Journal of Production Economics, vol. 106, no. 1, pp. 248-260, 2007.

[23] S. R. Singh and C. Singh, "Optimal ordering policy for decaying items with stock-dependent demand under inflation in a supply chain," International Review of Pure and Advanced Mathematics, vol. 1, pp. 31-39, 2008.

[24] S. R. Singh and S. Singh, "Two-warehouse partial backlogging inventory model for perishable products having exponential demand," International Journal of Mathematical Sciences and Computer, vol. 1, no. 1, pp. 229-236, 2008.

[25] S. R. Singh and R. Jain, "Evaluation of a practical inventory control and pricing policy for multivariable demand under inflation," Indian Journal of Mathematics and Mathematical Science, vol. 4, no. 1, pp. 67-77, 2008. 


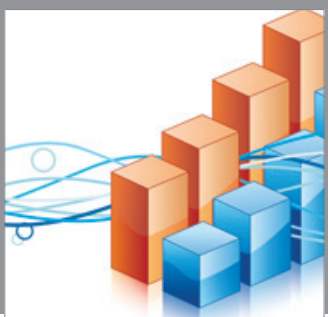

Advances in

Operations Research

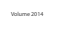

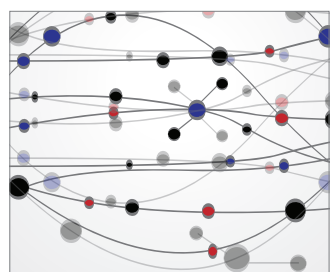

\section{The Scientific} World Journal
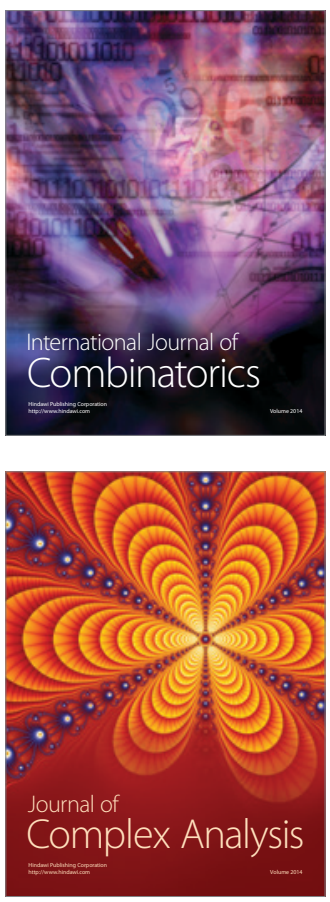

International Journal of

Mathematics and

Mathematical

Sciences
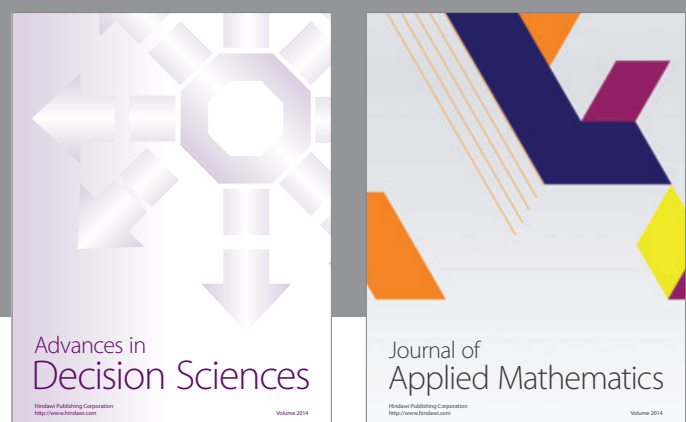

Journal of

Applied Mathematics
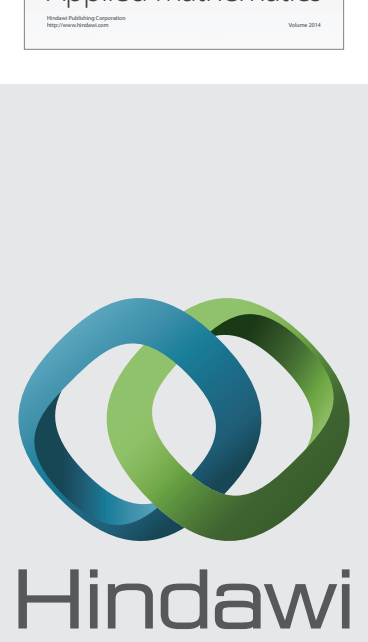

Submit your manuscripts at http://www.hindawi.com
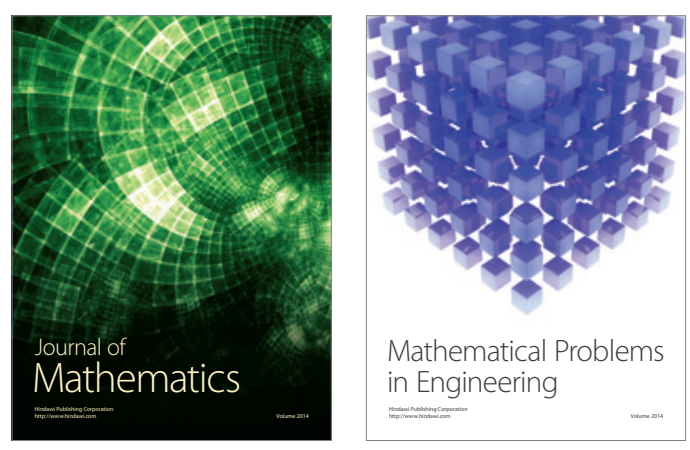

Mathematical Problems in Engineering
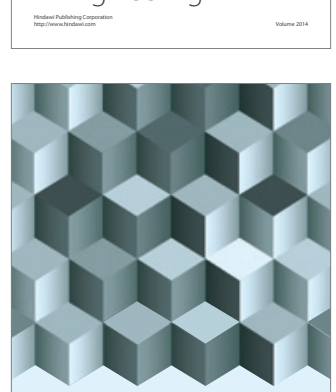

Journal of

Function Spaces
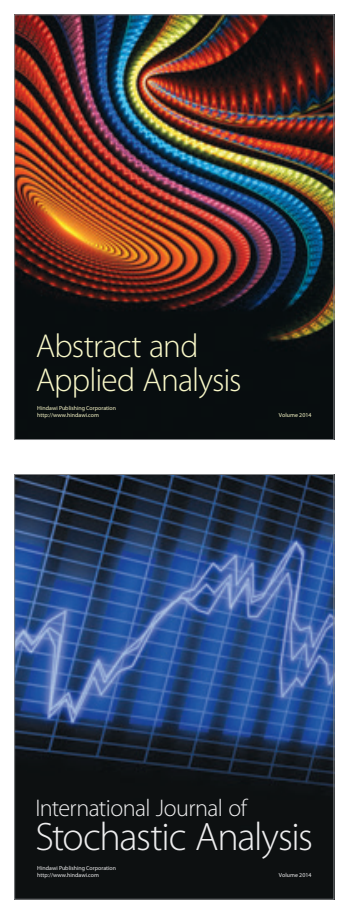

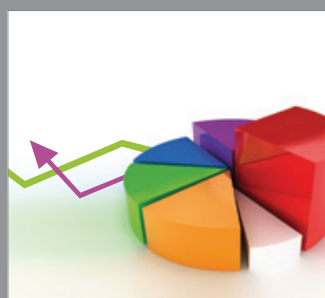

ournal of

Probability and Statistics

Promensencen
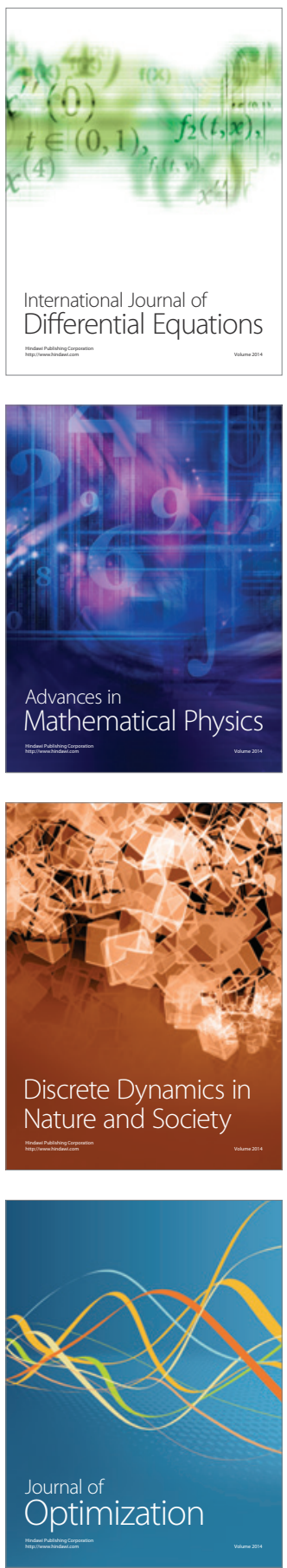